\title{
Screening: the age of fishes
}

\author{
Monya Baker
}

\begin{abstract}
Advances in microfluidics and imaging, combined with some high-profile studies, are increasing interest in whole-organism screening.
\end{abstract}

Randall Peterson did not enter graduate school planning to work with zebrafish. Instead he sought to understand how the immunosuppressant drug rapamycin interacts with mTOR, its mammalian protein target, now known to integrate cell-signaling pathways that control everything from cell motility to protein synthesis. In the months before receiving his degree, Peterson wanted to evaluate the physiological effects of rapamycin, and testing this on zebrafish larvae seemed a convenient way to do so. As both positive and negative controls, he tested various drugs. That is where his scientific career took a turn. "Each drug had a different and distinct developmental effect on the fish," he recalls. "I just knew this was going to be really cool."

Fish, along with other small, fast-developing organisms, offer a kind of middle ground between the ability to screen many molecules and to find molecules with physiological effects in vivo. Several organisms have been used for medium-throughput screens. These include the roundworm Caenorhabditis elegans, the zebrafish Danio rerio, the fruit fly Drosophila melanogaster and the African clawed frog Xenopus laevis (Box 1).

Compared with protein- and cell-based screening, whole-organism screening is slower and more expensive. Pharmaceutical companies typically maintain libraries of over a million compounds for screening, but whole-organism screening campaigns generally test hundreds or thousands of compounds, searching for those with physiological effects. Although understanding the mechanism of action for molecules identified in this way can be difficult, their potential relevance is clear: molecules identified as hits in organism-based screens have effects not just against a particular protein or cell type but also in a physiological

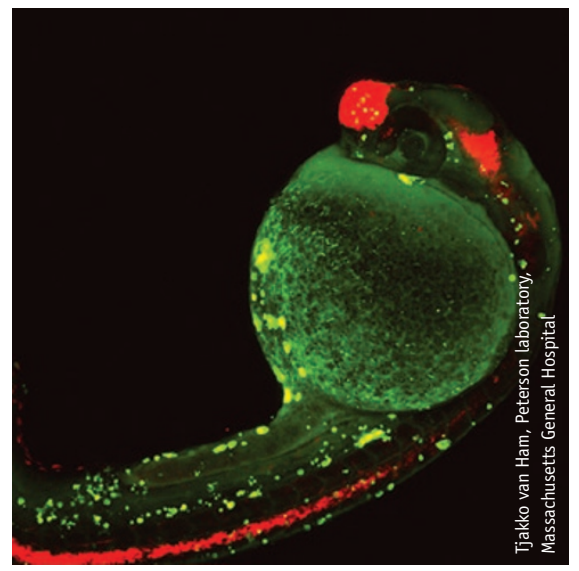

Organisms used in screens can be engineered so cells of interest display fluorescent markers. Neurons are shown in red and apoptotic cells in green.

context. Gene activity can be screened as well, allowing the organisms to be used for target validation and discovery.

Zebrafish larvae are most commonly used for whole-organism screens. Adult zebrafish are popular, too, but their mobility and larger size make them less convenient to use. Embryos develop quickly: within three days of fertilization a zebrafish has a vascular system, a beating heart, the fish equivalent of a pancreas and kidneys. Even better, the larvae, as well as some mutant adult strains, are transparent, facilitating imaging.

Findings from fish screens must still be verified in mammals, but evidence is accumulating that results often do translate to humans. As early as 2003, a study found that 22 of 23 drugs known to have a particular type of cardiotoxicity in humans also affected zebrafish heart rates ${ }^{1}$. At least one fish screening project has led to an experimental drug that is now in human clinical trials, and other identified molecules are being advanced toward this stage. Such progress is encouraging further work in the field, which is building a larger community of researchers well-versed in whole-organism screening as well as expanding technologies to make it faster and more informative.

\section{Suitable screening}

More important than bringing new technology into a screen is making sure that the screen is designed to answer desired questions, says Leonard Zon, who directs the Stem Cell Program at Children's Hospital of Boston, Harvard Medical School. A screen conducted in Zon's laboratory in 2007 led to identification of a molecule that is now in clinical trials.

The researchers incubated several thousand larvae with about 2,500 compounds already known to be biologically active. After staining the embryos for proteins associated with blood development, the researchers visually assessed the size and presence of 'purple dots' in their aortas, where blood first forms. Only about 80 compounds had detectable effects, but as the molecules were well characterized, this hit rate was enough to show clear patterns: compounds that led to increased synthesis of biomolecules known as prostaglandins also correlated with increased production of blood stem cells, whereas molecules that yielded decreased prostaglandin synthesis correlated with decreased numbers of blood stem cells ${ }^{2}$.

The researchers verified these effects in studies of mice and of human cord blood, and a molecule derived from this work is currently being tested for its potential to improve success of cord blood transplants in treating diseases such as lymphoma and leukemia. (Zon is a scientific founder of Fate Therapeutics, which is taking the project through clinical trials.) 


\section{BOX 1 FROM FISH TO FROGS}

Compared to fish, the value of frogs for whole-organism screening is vastly underappreciated, says André Brändli at Walter Brendel Centre of Experimental Medicine at the Ludwig Maximilians Universität, München. It is true that zebrafish, with their smaller size and faster generation times, offer certain practical advantages. However, he says, transparent frog embryos are available, and assessing phenotypes visually is convenient. And although producing enough zebrafish embryos for screening can be problematic, that is not the case for these amphibians:

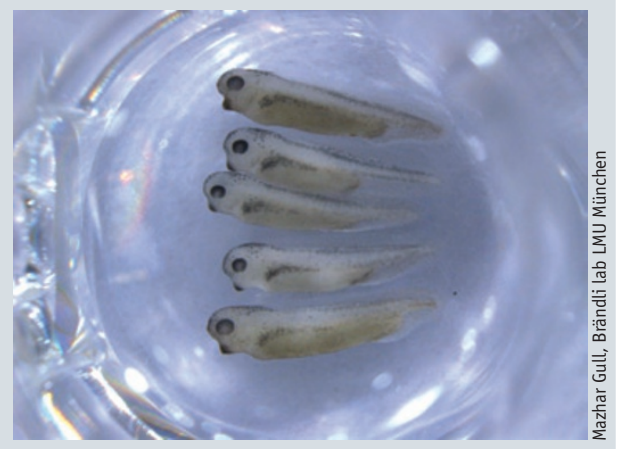

Two-day-old Xenopus laevis embryos arrayed in multiwell dishes for detecting compounds with desirable bioactivity. one female frog can lay a few thousand eggs at once, several times more than a pair of mated zebrafish. "I think it's a myth that frogs are more 'difficult' than fish," he says.

More important, Brändli says, is the fact that frogs are genetically closer to humans, branching off 360 million or so years ago as opposed to 450 million years ago. But Leonard Zon, who works with one of the world's largest zebrafish aquaria at Children's Hospital of Boston and has also worked with frogs, believes screens of both organisms can yield similar insights. "While amphibians are closer to humans, the gene set for organs is roughly the same for all vertebrates," he says. "The pancreas has insulin in all vertebrates. Blood cells have hemoglobin in all vertebrates." Still, Zon says, frogs can be a useful complement to fish.
In a separate effort led by Peterson, researchers screened 7,500 molecules in zebrafish larvae, looking for molecules that produce the same phenotype as genetic mutations that disrupt a signaling pathway involving bone morphogenetic protein (BMP). This screen led to identification of a compound not previously known to disrupt BMP signaling ${ }^{3}$. This compound is now used as a chemical tool for probing this pathway and is being evaluated in animal studies to treat a rare bone disorder.

Molecules identified in zebrafish screens will usually be reassessed in mice, so investigators need to compare the kind of information that can be gleaned from a fish screen with that obtained in simpler screens that also generate hits destined for mouse studies, says Cord Dohrmann, chief scientific officer of Evotec AG, which provides multiple screening services. Fish are already widely used for toxicity screens, particularly for liver development and heart function. Given the rate and extent of organogenesis in the maturing embryos, Dohrmann believes they could be particularly useful for regenerative studies, an area in which researchers lack extensive knowledge about targets and mechanisms that could be therapeutically useful.
And drug hunters would do well to remember that whole-organism screens are not the only kind of screening that is becoming more practical. Whereas zebrafish have about $70 \%$ of their genomes in common with humans, stem-cell technologies could provide fully human cells representing various tissues. Workers at Cellular Dynamics International, for example, differentiate cardiomyocytes from human induced pluripotent stem cells and supply them to pharmaceutical companies for drug testing for a wide variety of screens. Appropriate genetic engineering and follow-up studies can indicate whether a drug that decreases contraction is acting, for example, via structural elements or ion channels. Eventually, says Emile Nuwaysir, the company's chief operating officer, their products will not only represent human cells but also human genetic diversity. Next year, the company plans to launch a panel of cardiomyocytes representing several individuals of both genders. Although company scientists are still validating the technology, assessing drugs in human cells could provide information that studies in an intact but doublechambered zebrafish heart cannot.

Once researchers have decided to go with whole-organism screening, the key to designing a good screen is picking a suitable phenotype, says Zon. If a molecule restores a mutant phenotype to normalcy, for example, there is a good chance that it works within the mutated pathway. Fish can also be modified with transgenes to better represent human disease and to facilitate screens. (Fish expressing green, red and yellow fluorescent proteins are even sold commercially in some countries as pets.) In the summer of 2010, the Wellcome-Sanger Center released the latest zebrafish genome sequence assembly and declared that the project had reached a maintenance stage, and many researchers are hopeful that emerging techniques will allow tinkering with the native genome, an attractive alternative to inserting transgenes. These emerging techniques include zinc-finger technology being commercialized for research applications by Sigma-Aldrich and transposon-mediated

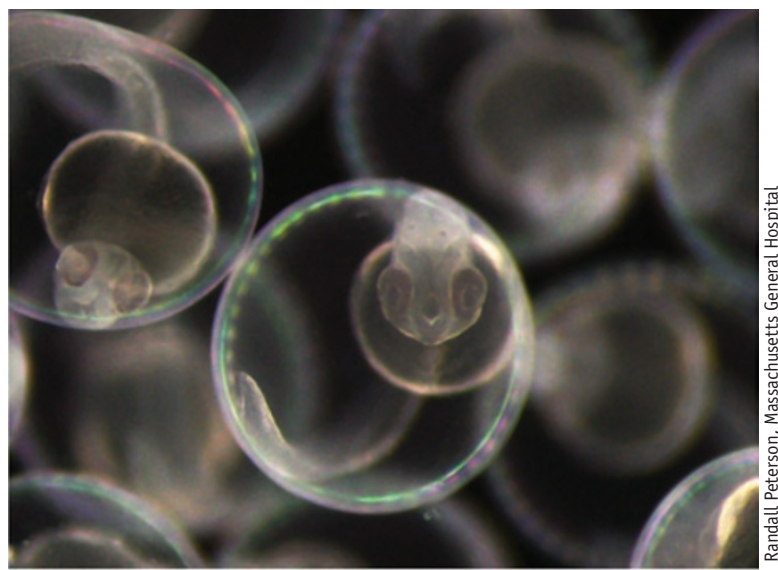

Whole-organism screens can reveal the effects of compounds in a physiological context. Only 30 hours after fertilization, zebrafish embryos' heads and tails are clearly developed. 
mutagenesis. Native genes can also be knocked down to some extent without genetic engineering, by injecting short stretches of chemically modified antisense DNA into early embryos.

Given the effort involved in wholeorganism screens, starting with molecules that have already been well characterized is probably the best option. Zon suggests starting screens with libraries such as the library of pharmacologically active compounds (LOPAC), which consists of well-characterized, biologically active molecules, before moving out to bigger, more diverse libraries, which could contain more-potent or additional classes of active molecules. That way, researchers can try to use the patterns of hits to figure out a mechanism. In some cases, researchers can chemically modify hits such that they can be used as tags to identify their protein targets, but doing so is often tricky and labor-intensive.

\section{Interpreting images}

The types of image-based screens used with zebrafish are limited only by researchers' imaginations, says Dohrmann. The amount of fluorescence in the insulin-producing beta cells can be quantified, for example, and even single-cell resolution is possible, he says.

But what is possible is not necessarily practical. For many scientists, the challenge is not in making sense of screens but in conducting screens sensibly. Most screens are assessed visually, which can be time-consuming. "The limiting thing for us is the scoring and analyzing of embryos," says Fabrizio Serluca, an investigator in the zebrafish group at the Novartis Institutes for Biomedical Research. "Automation: that's going to be the key."

Equipment designed for high-content screening of cells can be used to scan a 96-well plate of zebrafish in five to ten minutes, says Ahmad Yekta at GE Healthcare, referring to the latest version of his company's IN Cell Analyzer 2000. "It can capture the whole area of the well in one single picture, so you don't need multiple fields [to find the fish], and this is done on a resolution sufficient for most organ-level analysis," he says. In fact, because zebrafish embryos are thicker than cells, label-free imaging techniques are particularly suitable for this kind of imaging, he says, although fluorescence channels to observe transgenic proteins can be easily added. When higher resolution is needed, separate higher-magnification images can be taken, and accompanying software can stitch them together.

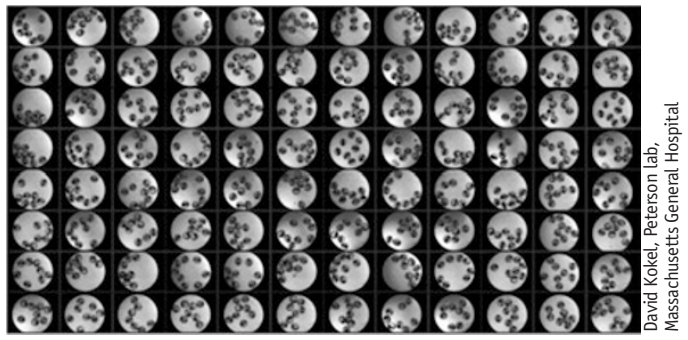

Zebrafish larvae are placed in a 96 -well plate for screening compounds.
However, automating image acquisition is not enough because that simply shifts the bottleneck to image analysis. In the first quarter of 2011, GE Healthcare plans to release software specifically designed to automate analysis of zebrafish imaging. "The difference between cells and zebrafish is that when you image the organs, they have a particular shape, so this took us to the idea of having an atlas that can be compared with real images," explains Yekta. Using images of dozens of wild-type fish, workers at the company developed a mathematical atlas that allows image-analysis software to find the fish and measure the size, shape and the fluorescence intensity of several organ regions. The software also calculates other parameters such as body curvature. Researchers will be able to adapt the software to identify and measure other features such as organ transparency, he says.

The product is still too new to have been compared head-to-head with manual image analysis in a high-throughput setting, says Yekta, but he expects that ongoing interaction with the zebrafish community will both extend and refine the product. There are some drawbacks. The machine itself costs around $\$ 200,000$, which is a major purchase for most laboratories. (The software, a plug-in to the IN Cell Investigator program that has not been priced yet, can be used independently of the machine.) For several parameters, the software works best for fish on their sides, but about a quarter of fish assume other orientations. The current system is also set up to analyze only one fish per well, and researchers often prefer to use three or more. Even so, Yekta believes appeal to many researchers conducting or considering zebrafish screens.

Researchers from the University of Pittsburgh recently described algorithms that automate image capture and analysis of the signal from fluorescent proteins in the heads of zebrafish embryos ${ }^{4}$. The technique is based on user-defined shapes and combines commercial products including the ImageXpress Ultra laser scanning confocal reader from Molecular Devices and software from Definiens Cognition Network Technology.

Other algorithms are enabling new categories of screens, says Peterson, now at Massachusetts General Hospital. "The human eye is pretty incredible at looking at shapes and patterns and identifying abnormalities, but there are quantitative measurements where image processing software is essential." High-throughput monitoring of how drugs change zebrafish heart rates, for example, would be impractical or impossible without automated imaging. And moresophisticated systems for watching behavior have allowed researchers to use zebrafish to find compounds with neurological effects. In one study, researchers incubated zebrafish embryos with 14,000 different compounds including known antidepressants, anesthetics and sedatives. They then exposed the embryos to a pulse of light and broke down each response into 14 parameters (such as when, whether and for how long the fish shook $)^{5}$. To analyze the screening data, researchers adapted clustering algorithms designed to analyze gene expression. These programs grouped molecules by their effects, that the ease, accuracy and throughput will

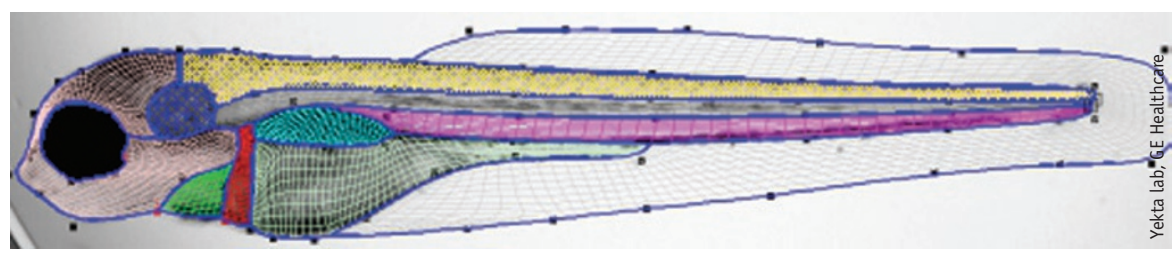

A digital atlas can be used to automatically find and measure several organs in zebrafish. 


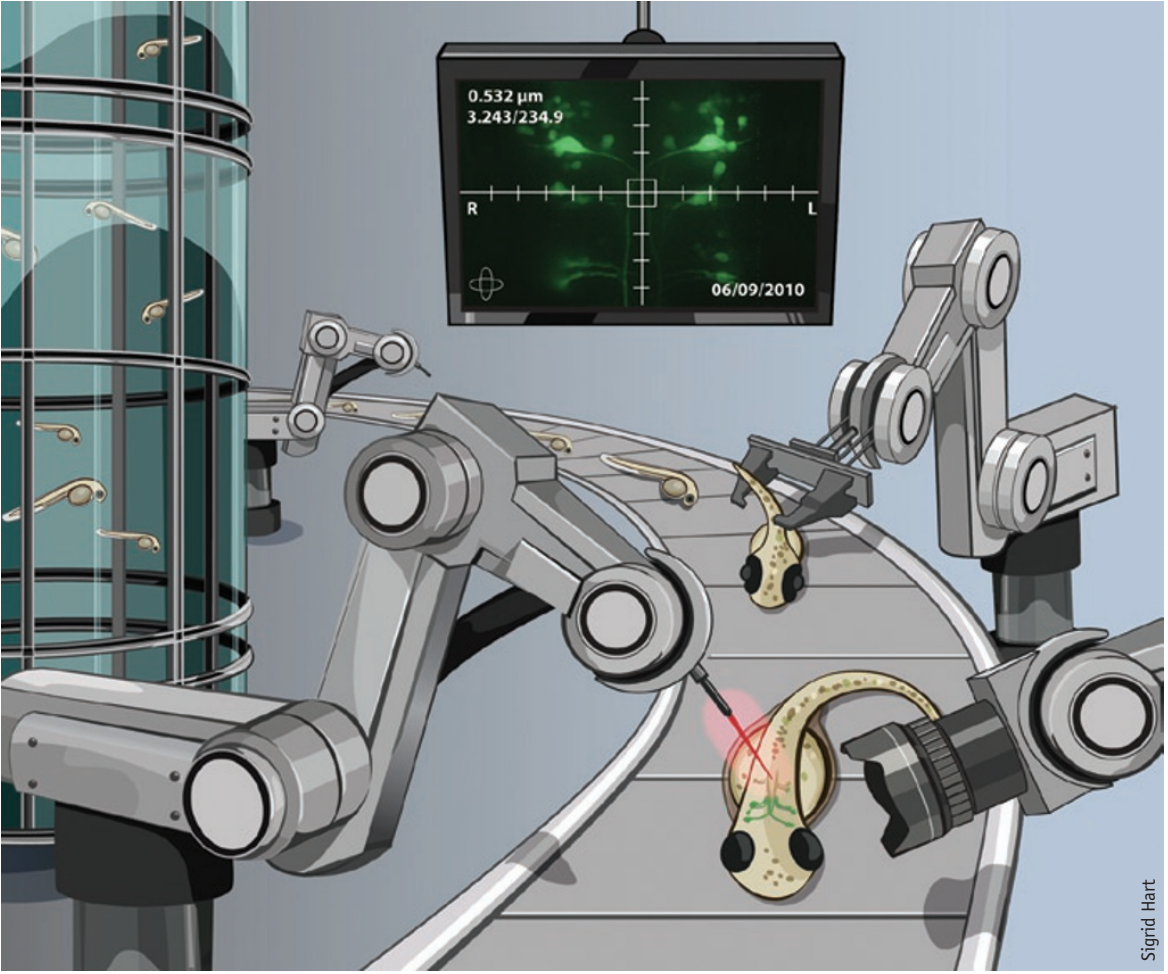

Microfluidics systems such as the one shown in this conceptual illustration of work from Mehmet Yanik's laboratory can automate manipulation and imaging in whole-organism screens.

allowing researchers to predict mechanisms of some uncharacterized compounds. In another study, researchers also took a clustering approach to look at about 4,000 compounds' effects on the rest-wake patterns of larvae and found, among other observations, that several anti-inflammatory compounds increased wakefulness ${ }^{6}$.

But simple physiology can thwart some of the most sophisticated imaging algorithms. Larvae and embryos are generally immobile and tend to lie on their sides, properties that enable image capture. However, if the fish orients itself on its back or belly, automated imaging programs may not be able to capture information from the brain or other organs, meaning that there is not enough data for subsequent analysis of a compound.

Some solutions to such complications are surprisingly low-tech. Using wells with round rather than flat bottoms can keep a fish in the center of a well rather than on the sides, where optics may be distorted. At least one company (Physical Sciences Inc.) makes wells designed so that fish can be imaged from the side as well as the top and bottom. However, these solutions are not panaceas. A light-scattering yolk sac and naturally occurring fluorescing molecules also complicate imaging. So does variation in body thickness and an inflating swim bladder.

\section{A microfluidics push}

Last year, Mehmet Yanik at the Massachusetts Institute of Technology described a microfluidics platform that can pull a fish from a well into a tiny chamber, where the fish can be rotated into a desired orientation, have its organs imaged with a confocal microscope and then be moved back into the well ${ }^{7}$. In that work, each image took less than 20 seconds to collect ${ }^{7}$, but even at that pace, screening a 96-well plate with one fish per well takes over half an hour. However, Yanik says that the system could be improved to run screens in parallel and that the time to take each image has already been cut in half. Yanik has launched a company around this technology, called Entera Pharmaceuticals, which is being funded by large pharmaceutical companies to conduct several zebrafish screens on million-compound libraries, he says. That task is expected to take a year or more.

Moving fish in and out of wells adds time to screens, but for some applications the investment is well worth it, says Novartis's Serluca, whose company is investigating the technology. "If we're looking at a very small structure like a pancreatic islet, it would be much tougher to judge by eye than with a computer even with the time required to load the fish [into an imaging chamber] and take the image," he says.
Microfluidics can also be used for other manipulations and for other organisms. Yanik has already developed systems for C. elegans, one of which performs microlaser surgery on nerve axons, allowing researchers to study regeneration. Hang Lu of Georgia Institute of Technology has also developed systems that combine microfluidics with imaging, focusing on D. melanogaster embryos and C. elegans. She and others have developed microfluidics systems that move a worm into place, immobilize it for imaging and even sort the worms by phenotype. Automated imaging allows researchers to make quantitative rather than qualitative assessments of parameters such as worm movementsomething manual techniques are often too variable to do. The human eye is very good at pattern detection, she says, but it performs less well when, as is often the case with these systems, the organisms cannot be examined side by side. For subtle differences, such as slight differences in size or brightness, computers can give much more quantitative data, she says.

But both the microfluidics and imaging aspects for whole-organism phenotyping are still very much developing technologies, Lu says, and technology developers will need to be careful how they evaluate robustness when designing systems for high-throughput applications. "Being able to process one or ten samples is not the same as processing a thousand samples," she says. When considering whether a system will help biologists, she says, it is not

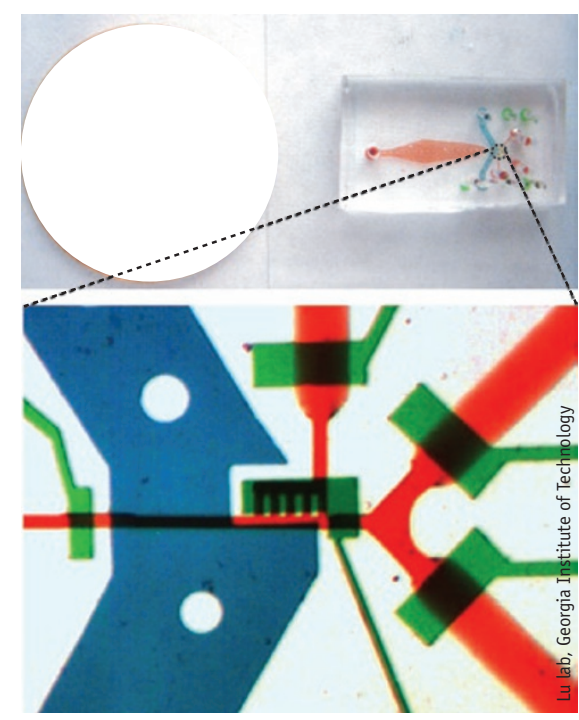

A penny-sized microfluidics machine can quickly immobilize, examine and sort worms according to desired phenotype. 
appropriate to calculate throughput by multiplying the number of samples by how long a platform takes to run one sample. Parameters such as how long the machine can run at a stretch, and how often a sample run fails must also be considered.

Ultimately, though, winning biologists over to whole-organism screening may depend less on technological innovations themselves than on the biological breakthroughs credited to screening.
That, says Peterson, takes time. "You see thousands and thousands of fish and then you happen to look into a well and you are so startled by what you see. The Eureka moment, if you have to encapsulate it, is in that moment," he says. "The follow-up is where the real work is, and that happens in months or years."

1. Milan, D.J., Peterson, T.A., Ruskin, J.N., Peterson, R.T. \& MacRae, C.A. Circulation 107, 1355-1358 (2003).
2. North, T.E. et al. Nature 447, 1007-1011 (2007).

3. Yu, P.B. et al. Nat. Chem. Biol. 4, 33-41 (2008).

4. Vogt, A., Codore, H., Day, B.W., Hukriede, N.A. \& Tsang, M. J. Vis. Exp. 40, 1900 (2010).

5. Kokel, D. et al. Nat. Chem. Biol. 6, 231-237 (2010).

6. Rihel, J. et al. Science 327, 348-351 (2010).

7. Pardo-Martin, C. et al. Nat. Methods 7, 634-636 (2010).

\section{Monya Baker is technology editor for \\ Nature and Nature Methods \\ (m.baker@us.nature.com).}

\begin{tabular}{|c|}
\hline Web address \\
\hline http://www.aquatichabitats.com/ \\
\hline http://www.ahdiag.com/ \\
\hline http://www.andor.com/ \\
\hline http://www.zeiss.com/ \\
\hline http://www.definiens.com/ \\
\hline http://www.fatetherapeutics.com/ \\
\hline https://www2.gehealthcare.com/ \\
\hline http://www.leica-microsystems.com/ \\
\hline http://www.lifetechnologies.com/ \\
\hline http://www.olympusamerica.com/ \\
\hline http://las.perkinelmer.com/ \\
\hline http://www.phylonix.com/ \\
\hline http://www.psicorp.com/ \\
\hline http://www.roche.com/ \\
\hline http://www.scanbur.eu/ \\
\hline http://www.segrestfarms.com/ \\
\hline http://www.sigmaaldrich.com/ \\
\hline http://www.thermofisher.com/ \\
\hline http://www.unionbio.com/ \\
\hline
\end{tabular}

